\title{
DIE FLEDERMAUS IN RELIGION, BRAUCHTUM UND KUNST INDONESIENS UND SEINER NACHBARGEBIETE
}

\author{
Von Alfred SteinmanN
}

Mit 7 Abbildungen

Vor Jahresfrist hat P. WIRZ ${ }^{1}$ in dieser Zeitschrift eine Abhandlung über die Rolle veröffentlicht, welche die Fledermaus in den religiösen und abergläubischen Vorstellungen sowie in der Kunst verschiedener Völker - und dabei speziell in derjenigen Chinas und der Hochkulturvölker Mittelamerikas - gespielt hat bzw. noch spielt. Diese seine Ausführungen gaben uns Veranlassung, ergänzend auf die Bedeutung der Fledermaus bei einigen Völkern und in einigen Gebieten hinzuweisen, die in der oben genannten Untersuchung keine oder eine nur unzureichende Würdigung und Berücksichtigung gefunden haben. Dies betrifft in erster Linie Indonesien, Melanesien, Australien und schließlich auch China.

Die Bedeutung, die der Fledermaus in den religiösen und mythologischen Vorstellungen der Völker Indonesiens zukommt, beschränkt sich im wesentlichen auf die Funktion, die sie vor allem im Bereich der Orakeldeutung, des Totemismus, im Jenseitsglauben und in den Schöpfungsmythen ausübt.

In der indonesischen Orakeldeutung tritt die Fledermaus als Omentier in Erscheinung, und zwar vorwiegend im östlichen Teil des Archipels, wo sie zum Beispiel auf Ceram, Celebes und Timor ihres unheilbringenden Wesens und Charakters wegen gefürchtet und gemieden wird. Wie aus den Beobachtungen ADAms ${ }^{2}$ und KruYrs ${ }^{3}$ hervorgeht, ist ihr unheilvoller Einfluß auf Celebes mit dem dortigen Vampirglauben verknüpft. Während im Gebiete der Minahassa (Residenz Menado, Nordost-Celebes) nach ADAM die Vorstellung, daß sich Vampire in Fledermäusen inkarnieren bzw. verwandeln können, verbreitet ist, konnte ich einer brieflichen Mitteilung von Dr. ALB. KRUYT entnehmen, daß unter den verschiedenen, im Archipel einheimischen Fledermausarten bei den Toradjas in Zentral-Celebes vor allem der große, als "Fliegender Hund» oder "Flughund» bezeichnete Pteropus (Mal: Kalong, Celeb: Paniki) im Geistesleben, in der Vorstellungswelt eine besondere Rolle spielt, während die gewöhnlichen, scharenweise in den Höhlen, in denen die Toradjas die Gebeine ihrer Toten auszusetzen pflegen, auftretenden kleinen Fledermäuse dagegen weder im Glauben noch in den zahlreichen Tierfabeln irgendwie erwähnt werden. Der Kalong gilt dort als Erscheinungsform einer vampirartigen Hexe, deren Schrei oder Ruf der Bevölkerung Angst und Schrecken einflößt.

Aus der verschiedenartigen Intonation ihres Schreies pflegt man sogar zu erkennen, ob sie ein menschliches Opfer eingefangen hat. Mit dem Ausruf "Hier sind Dornen» versucht man diese Fledermaushexe fernzuhalten in der Meinung, da $\beta$ sie aus Furcht, sich an den Dornen zu verwunden, die Bewohner ungeschoren läßt. Auch sollen die als blutsaugende Vampire umherirrenden und besonders gefürchteten Seelen der bei der Geburt eines Kindes gestorbenen Frauen (sogenannte Punti-anak) mitunter in Gestalt eines Fliegenden Hundes in den Leib von Schwangeren eingehen und die Gebärorgane durch ihren $\mathrm{Bi} \beta$ vernichten.

$\mathrm{Zu}$ den auf der Insel Ceram als unglückbringend verschrienen Omentieren soll nach VALENTIJN ${ }^{4}$ außer den Eulen und einigen anderen Unglücksvögeln (Manu «k»

1 Paul WIRz: Uber die Bedeutung der Fledermaus in Kunst, Religion und Aberglauben der Völker. Geographica Helvetica, III, 1948, 3, S. 267-278.

${ }^{2}$ L. ADAM: Zeden en gewoonten en het daarmede samenhangend adatrecht van het Minahassa'sche volk. Bijdragen, deel 81,1925, S. 480 .

3 Ali. C. KRUYT: De Timoreezen. Bijdragen, deel 79, 1923, S. 434.

4 F. Valentijn: Oud en nieuw Oost-Indien. Dordrecht 1724-1726, II, 1., S. 81, zitiert bei E. StresemanN. Religiöse Gebräuche auf Seran. T.B.G. deel 62, 1923, S. 315. 
salewane) auch die kleine Fledermaus gẹören; es wird dort als ein für den Kopfjäger sehr schlimmes Vorzeichen angesehen, wenn eine niedrig fliegende Fledermaus auf ihn zukommt, über ihn hinwegfliegt und wieder umkehrt. Auch auf der Insel Timor gilt es als ungünstiges Vorzeichen, wenn ein Krieger auf seinem Vormarsch in der Dämmerung mit einer umherflatternden Fledermaus in Berührung kommt. Sie ist dort nicht nur ein schlimmes Omenzeichen, sondern spielt auch bei religiösen Vorstellungen eine Rolle. Die Fledermaus kann bei den Timoresen übrigens auch mit dem Jenseitsglauben verknüpft sein, wo sie als eine "Ban-fan» genannte Wächterin der Himmelspforte auftritt, deren Aufgabe darin besteht, die eintreffenden Seelen nach dem auf Erden von ihnen begangenen Unrecht zu befragen und für die Abbüßung ihrer Strafe zum Himmelsherrn Usif neno zu bringen; doch ist nicht sie selbst, sondern der Sonnenhund Asu neno der eigentliche Wegweiser und Seelenführer. Abgesehen von Timor, spielt die Fledermaus im übrigen Indonesien, soweit mir bekannt ist, weder in den Jenseitsvorstellungen noch in den überaus zahlreichen Tierfabeln eine Rolle. Damit dürfte auch die Feststellung, daß im Brauchtum der indonesischen Völker der Fledermaus eine relativ geringe Bedeutung zukommt, übereinstimmen. Zwar wird nach KRUYT ${ }^{5}$ heute noch auf der kleinen Insel Mentawei (Westindonesien) bei besonderen Gelegenheiten unter den im "Uma»-Stammhaus stattfindenden Tänzen, welche die Bewegungen von Tieren nachahmen, durch das Haupt der Dorfgemeinschaft, den sogenannten Rimata, persönlich der als "Turuleituak» ' bezeichnete "große Fledermaustanz» ausgeführt; doch dient dieser nach einer persönlichen Mitteilung von Dr. KrUYT ursprünglich wohl als Jagdzauber (zur Sicherung des Erfolges bei der Fledermausjagd) gedachte Tanz heute im allgemeinen nur noch als eine Art Volksbelustigung.

Ungleich wichtiger und interessanter ist jedoch wohl die Rolle, die der Fledermaus in Indonesien als Totemtier zukommt: Es sind wiederum einige Inseln im östlichen Indonesien, auf denen sowohl die eigentliche Fledermaus wie der Fliegende Hund (Pteropus) in dieser Eigenschaft in Erscheinung treten. Unter diesen Inseln ist Ostflores zu nennen, wo sich nach E. VATTER ${ }^{7}$ eine bestimmte Tabuvorschrift im Dorf Lewo Tobi für die Frauen der Kledangsippe (außer auf den Fisch) auch auf den Fliegenden Hund bezieht. Im östlichen Teil der Insel Adonare ist es dagegen nach P. ARNDT ${ }^{8}$ eine kleine Fledermausart, für die bei den Clans Onge nulan und Bahi ein Speiseverbot besteht; bei Übertretung dieser Tabuvorschrift würden die Leute des erstgenannten Clans von Krankheit heimgesucht werden, im Bahi-clan dagegen die Kinder Gesichter wie diejenigen der gegessenen Tiere bekommen. Auf der Insel Solor spielt die Fledermaus außerdem noch in der Ursprungsmythe des Clans Lewo Hajong als Helferin eine Rolle, indem sie zusammen mit anderen Tieren der Stammutter beim Bestehen verschiedener, ihr von ihrem Vater Laga Doni auferlegten Prüfungen hilfreich zur Seite gestanden haben soll, und zwar habe sie das von Laga Doni ins Meer geschüttete Erdöl, das seine Tochter, das heißt die Stammutter des Lewo Hajong-clans, in einem Faß zurückbringen sollte, für sie herausgeholt.

Es ist vielleicht kein Zufall, daß die Fledermaus ausgerechnet im östlichen Teil Indonesiens als Totemtier in Erscheinung tritt. Die Vermutung, daß hier Einfüsse und Beziehungen der benachbarten Gebiete, wie Melanesien und Australien, vorliegen, ist naheliegend. Auf der Insel Vuatam (Watom) im Bismarck-Archipel ist nach C. LAUFER ${ }^{9}$ der Fliegende Hund (zusammen mit dem Dugong und dem Fischadler) das Totemtier der Vunamakadao-Heiratsklasse. Im benachbarten Australien dagegen

${ }^{5}$ Alb. C. KRuYt: De Mentawaiers. T.B.G. 1923, S. 73.

- Im Gegensatz zu den kleinen «Regu-regu»-Fledermäusen.

7 E. VAtTer: Ata Kiwan, 1932, S. 150.

${ }^{8}$ P. ARndT: Soziale Verhältnisse auf Ostflores, Adonare und Solor. Internationale Sammlung ethnographischer Monographien, Bd. IV, Heft 2, 1940, S. 151, 153, 217.

9 C. LAufer: Der Fliegende Hund im Brauchtum der Qunantuna auf Neubritannien. Acta tropica, V. 3, 1948, S. 228-243. 
gilt die Fledermaus nicht nur im ältesten Teil bei einigen Kulin-Stämmen (Südostaustralien) und an der Ostküste, bei den Chepara und dem Port-Stephens-Stamm sowie in Westvictoria, sondern auch bei den Yuin-kuri, Wiradyuri-Kamilaroi und anderen Stämmen im Norden und Nordwesten als männliches Geschlechtstotemtier.

Im Wohngebiet jener Kulin-Stämme, wo die Fledermaus als überall gleiches Totemtier neben irgendeinem anderen weiblichen Geschlechtstotem auftritt, findet auch bei den Knabeninitiationsfeiern durch Ausschlagen eines oder beider oberer Schneidezähne eine Angleichung an den (Fledermaus)Stammvater statt. Im nördlichen und nordwestlichen Teil Australiens, wo die stumme, stimmlose Fledermaus ebenfalls als männliches Geschlechtstotemtier gilt, erfolgt die Angleichung der Novizen bei den Initiationsfesten durch Nachahmung der Stummheit (Verständigung im Lager nur durch Zeichen) und durch die besondere Körperhaltung mit straff an den Seiten gelegten Armen und Händen, wodurch die Stellung des Tieres mit eingezogenen Flughäuten in Ruhelage angedeutet werden soll. Auch im Gebiet von Port-Stephen und von Westvictoria gehört die Fledermaus (neben der Eule, dem Nachtfalken, dem Baumläufer und dem Ziegemelker) zu den Nachtvögeln, die das Geschlechtstotempaar bilden.

Nicht nur auf der ostindonesischen Insel Solor, sondern auch in Australien ist die Fledermaus mit verschiedenen Ursprungsmythen verknüpft.

Nach einer auch von WIRz zitierten Mythe der Eingeborenen von Lake Condal in Victoria 10 über den Ursprung des Feuers gilt sie als Stammvater der Menschen, und bei den Wotjobaluk (Südostaustralien) wird die Entstehung des Menschengeschlechts ebenfalls auf sie zurückgeführt. Zudem gilt sie dort als Bringerin des Feuers, das sie durch Reiben zweier Hölzer entfacht hat ${ }^{11}$.

Auch in der Schöpfungsmythe der Qunantuna von Neubritannien spielt die Fledermaus, i. b. der Flughund Pteropus, nach LAUfer als das erste vom Urgeist nach Erschaffung des ersten Menschen der ersten Frau in den Schoß gelegte Tier eine Rolle. Da der Flughund bei den dortigen Eingeborenen als Sinnbild der Geschlechtlichkeit gilt, symbolisiert diese Handlung die Erschaffung der äußeren weiblichen Sexualorgane.

Der bereits obenerwähnten Untersuchung LAUFERs ist $\mathrm{zu}$ entnehmen, daß die Fledermäuse bei den Qunantuna auf Neubritannien als Inkarnationen Verstorbener, als Geistertiere gelten. Die Mitglieder der dortigen Männergeheimbünde, denen die Fledermaus im Traum als Schutzgeist zu erscheinen pflegt, glauben, daß sie sich nach dem Tode in Vögel oder in Fliegende Hunde verwandeln, die in den kühlen, schattigen Ficusbäumen ein ruhig-beschauliches Dasein führen. Ihre Sammelplätze (Ficusbäume, Höhlen und Schluchten) sind dann auch die Eingänge ins Totenreich. Infolge ihrer bekannten Zählebigkeit ist die Fledermaus auch zum Sinnbild der Unsterblichkeit geworden. Ähnlich wie in Insel-Melanesien, wo der Flughund in der Schöpfungsmythe der Qunantuna (Neubritannien) eine Rolle spielt, tritt nach WIRz der Fliegende Hund auch in der Mythologie und in der Kunst der Marind-anim von Holländisch-Südneuguinea in Erscheinung. Auf Rindenstoffen werden oft in schwarz-rot-gelber Malerei Fledermaustänzer
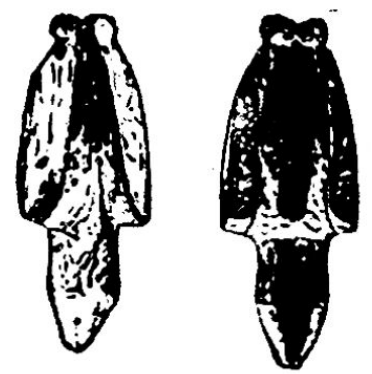

Fig. 1. Holzskulptur des Flughundes . Pteropus (hängend, Schlafstellung) aus dem Tempel vonLembongpangi (Celebes). Sammlung Sarasin. (Nach MEYer und Richter) dargestellt. (Siehe auch Abbildung bei WIRz, P., Die Marindanim von Holländisch-Südneuguinea, III, Tafel 17, Abb. 2, IV, Tafel 33, Abb. 1, und Tafel 34, Hamburg 1925.) Trotzdem die Fledermaus, wie aus dem Vorhergehenden hervorgeht, in den religiösen Vorstellungen, im Jenseitsglauben, in der Orakeldeutung und im Totemismus keinen unwichtigen Platz einnimmt, tritt sie in der indonesischen Kunst als Verzierungsmotiv relativ selten in Erscheinung, und ihre Darstellung steht merkwürdigerweise in keinerlei Beziehung zu ihrer sonstigen vielseitigen Bedeutung! Unter den wenigen mir bekannt gewordenen indonesischen Fledermausdarstellungen sei hier die in Kerbschnitt auf einer Holzplanke angefertigte Abbildung eines Fliegenden Hundes aus der westindonesischen Insel En g g a n o erwähnt, über deren Bedeutung jedoch

10 B. Smyth: The aboriginal of Victoria. London 1871, II, S. 462.

11 A. W. Howrrt: The native tribes of South-East Australia, London, S. 484. 
nichts Näheres bekannt ist ${ }^{12}$. Eine weitere, ursprünglich von MEYer und RICHTER ${ }^{13}$ stammende hölzerne Darstellung des Fliegenden Hundes (Pteropus) auf Celebes aus der Sammlung Sarasin, die als Sitz des Dorfgeistes gelten soll (Fig. 1), nach KruYTs Auffassung jedoch eher ein Abwehrmittel gegen fledermausgestaltige Hexen sein dürfte, beweist, daß derartige, offenbar recht ungewöhnliche Darstellungen in alteinheimischen Vorstellungen wurzeln. Abgesehen von diesen Ausnahmefällen, wird man in der An-

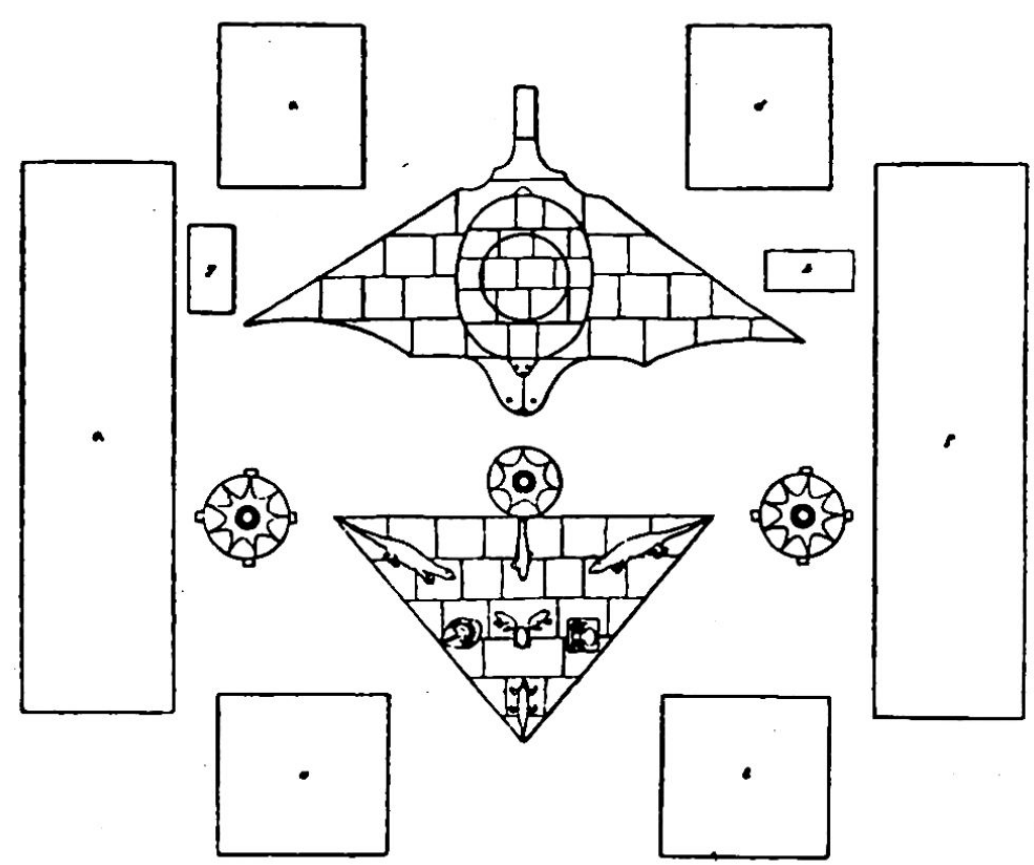

Fig. 2a. Grundriß der achten Terrasse des Heiligtums Tjandi Tjeto in Zentraljava. (Nach M. Schnitger) nahme, daß die meisten plastischen Darstellungen der Fledermaus in der darstellenden Kunst Indonesiens zweifellos mit dem Einfluß der Hochkulturen und dabei vor allem Chinas im Zusammenhang stehen, kaum fehlgehen. Als Beispiel sei auf die in ihrer Art einzig dastehende steinerne Fledermausdarstellung des berühmten Terrassenheiligtums von $T j$ e to in Zentraljava aus dem 15. Jahrhundert hingewiesen, auf dessen achten Terrasse sich die wohl älteste und mit Sicherheit datierbare Wiedergabe einer riesigen Fledermaus befindet. Wie aus dem abgebildeten Grundriß (Fig. 2a) hervorgeht, trägt das mit ausgebreiteten Flughäuten flach auf dem Boden liegend dargestellte Tier auf seinem Rücken eine Schildkröte (Fig. 2b). Vor dem Kopf der Fledermaus liegen drei runde, flache Steine mit sternförmigen Figuren, und in der Mitte befindet sich ein dreieckiges Gebilde mit neun als sexuelle bzw. Fruchtbarkeitssymbole bekannten Wassertieren. Die Kombination derFledermaus mit einer Schildkröte und die übrigen Tiersymbole bilden für die Archäologen heute noch ein ungelöstes ikonographisches Rätsel, über dessen Deutung und Bedeutung verschiedene Theorien aufgestellt worden sind ${ }^{\mathbf{1 4}}$.

Während man vielfach aus der Uberlegung heraus, daß die Fledermaus heute noch als ein der Göttin Durga geweihtes Tier gilt, diese Darstellung mit einem alten Fledermauskult in Zusammenhang zu bringen suchte, hat $C_{R \cup C Q}$ auf eine andere, nicht minder interessante Erklärungsmöglichkeit hingewiesen, die wir hier lediglich erwähnen möchten, ohne gleichzeitig irgendwie dazu Stellung zu nehmen. CRUCQ glaubt nämlich, im steinernen Dreieck ein kosmisch-sexuelles Erlösungssymbol und in den damit zusammenhängenden Tierdarstellungen eines jener altjavanischen Bildchronogramme (sogenannte Tjandra-sengkala) zur Andeutung einer nur den Eingeweihten bekannten Jahreszahl erkennen zu können, dessen Enträtselung die Jahreszahl 1373 Çaka (1451 n. Chr.) zu ergeben scheint, die möglicherweise das Stiftungsdatum des fürstlichen Heiligtums darstellt. Die Datierung nach der Çaka-aera beginnt mit dem Jahr $78 \mathrm{n}$. Chr. Was nun die Deutung der Kombination Fledermaus-Schildkröte betrifft, so lautet seine, vorläufig als Arbeitshypothese zu bewertende Auffassung dahin, daß ähnlich wie in China, wo die Bedeutung der Fledermaus als Glücks- und Reichtumssymbol auf dem Gleichklang des Wortes «Fu» (=- Glück) beruht, auch hier wegen der Analogie der Wörter Lawah (= Fledermaus) und Lwah (= Wasser) die Fledermaus als Sinnbild des Ozeans, des Wassers, aufzufassen.sei, in welchem die Schildkröte, die bekanntlich auch Vishnus Avatara darstellt, den als Quirlstock dienenden Berg Mandara stütze. Diese Bildkombination würde also nach CrucQs Auffassung die aus dem Quirlen des Ozeans her-

${ }^{12}$ A. KRaEmer: Westindonesien, 1927, Stuttgart, Tafel XVII, Nr. 23.

${ }^{13}$ Siehe W. Kaudern: Art in Central Celebes. Göteborg, 1944, Fig. 239, S. 377.

${ }^{14}$ K.C.CrucQ: De figuren op het achtste terras van Tjandi Tjeta.Tydsch. Bat.Gen.1933, deel 73, S.151-155. 


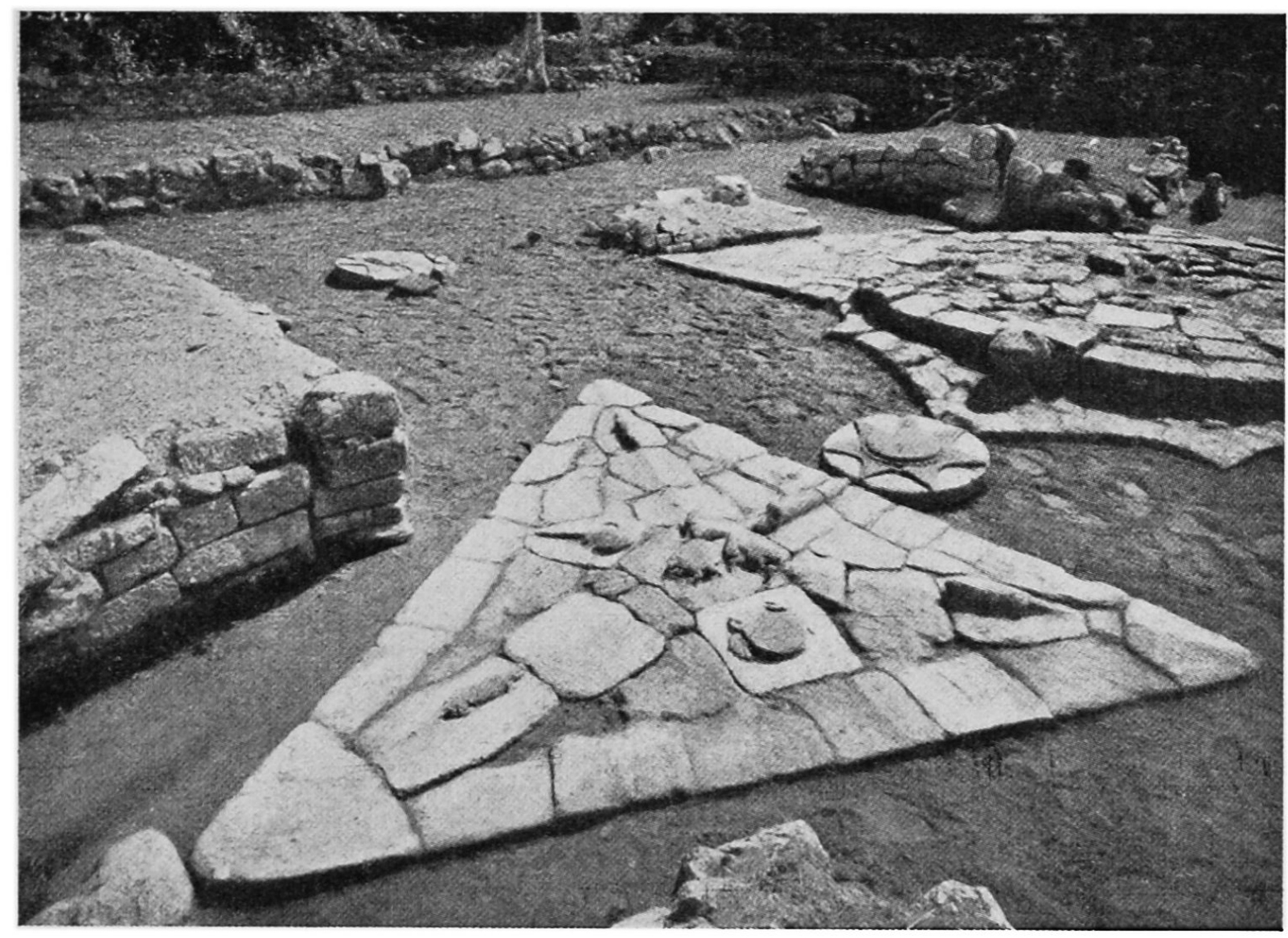

Fig. 2b. Blick auf die achte Terrasse des Heiligtums Tjandi Tjeto in Zentraljava mit der Schildkröte im Hintergrund rechts. (Nach R. C. CRUCQ)

vorgehende Entstehung des Unsterblichkeitstrankes (Amrita) symbolisieren, was in einem Heiligtum, in welchem das Erlösungswasser eine wichtige Rolle spielt, durchaus verständlich wäre und womit auch das Vorkommen von Wassertieren in Einklang stünde.

In der alten Hindu-Kunst von Java ist, soviel mir bekannt ist, die Fledermaus als Ziermotiv nirgends anwesend; dagegen befinden sich unter den steinernen Basreliefstücken in hindujavanischem Stil aus der Mitte des 16. Jahrhunderts, die als Rosetten, Medaillons, Randverzierungen usw. die Wände des restaurierten Gebäudekomplexes der Grabstätte des Fürsten Ratu Kalinjamat Mantingan südlich von Japara als Dekoration zieren, zahlreiche Fledermausdarstellungen. Sie stammen aus der alten Moschee, die nach einem dort aufgefundenen und entzifferten Bildchronogramm oder Tjandra-sengkala die Jahrzahl 1559 (oder 1481 Çaka) angibt. Kunsthistorisch wichtig sind diese kleineren und größeren Steinornamente mit ihren eigenartigen, der hindujavanischen Kunst entlehnten Motiven vor allem als typische Exponenten eines besonderen Kunststils, nämlich desjenigen aus der Übergangszeit von der hindujavanischen zur mohammedanischen Epoche ${ }^{15}$. Das Fledermausmotiv tritt hier in der Regel als Eckverzierung der unter den Steinornamenten am häufigsten vorkommenden runden Medaillons von 35-37 cm Durchmesser auf, und es kann wohl kein Zweifel darüber bestehen, daß hier chinesis che Einflüsse vorliegen! Die Verwendung der mehr oder weniger stilisierten Fledermaus in Form von vier Eckverzierungen eines zentralen runden Medaillons ist in China sehr häufig. Lessing ${ }^{16}$, der für China nur die symbolische Kombination von fünf Fledermäusen erwähnt, scheint ihr Vorkommen in der Vierzahl übersehen zu haben, vielleicht deshalb, weil diese Anordnung weniger symbolischen als vielmehr orna-

15 Bericht von Th. C. Leeuwendal (Ass.-Resident in Japara) in: OudheidkundigVerslag 1930, S. 52-57.

${ }^{16}$ F. Lessing: Úber die Symbolsprache in der chinesischen Kunst. Sinica, IX, Heft 3/4, S. $142,1934$. 
mental-dekorativen Charakter hat. Als Beispiel sei auf die bei MüNSTERBERG ${ }^{17}$ abgebildeten Deckenkassetten aus den Grabtempeln der Mandschu-Kaiser in Siling aus dem 18. und 19. Jahrhundert (1723-1851) hingewiesen; auf zahlreichen chinesischen Buntstickereien auf Seide, auf Schnitzereien, welche die Deckplatte chinesischer Himmelbetten verzieren, sowie auf zahlreichen anderen Gegenständen treten immer wieder die vier eckständigen Fledermäuse auf. Aber auch sonst zeigen die Basreliefs von Mantingan im nördlichen Teil von Java noch andere, auf chinesische Beeinflussung hinweisende Merkmale, unter denen hier nur die mit dem Yui-Szepter-Wolkenmotiv kombinierte Lotusblumenstilisierung, die Kalebasse usw. erwähnt seien ${ }^{18}$.

Recht interessant und aufschlußreich sind auch die Fledermausverzierungen in den zahlreichen chines is chen Tempeln (Klenteng) auf Ja va und anderen Inseln des Malaiischen Archipels. Die von J. BRANDES ${ }^{19}$ darüber veröffentlichte stilkritische Untersuchung bringt ganz überraschende Hinweise auf die teilweise Umbildung des alten, als Kala-Makara (Fischelefant) bekannten Hindu-Ornaments zu fledermausartigen Formen und Motiven.

Bei der Erörterung der dabei zutage tretenden eigenartigen Stiltendenzen hebt BRANDES die Fledermausdarstellungen mit ihren unendlich-mannigfachen Varianten im Klenteng von Malang in Ostjava hervor, deren Anordnung und Gruppierung besonders charakteristisch ist. Dort sind die Makaras nämlich oft derart angeordnet, daß sie zusammen einen Löwenkopf bilden; diesem gibt man wiederum die Gestalt einer Fledermaus, und dasselbe geschieht mit den am meisten in den Vordergrund tretenden Partien der Makaras ${ }^{20}$. Das Einfügen stilisierter Fledermausmotive in die Kala-Makara-Ornamente (von den Chinesen "Sjiho-Stücke» genannt) bringt BRANDES mit der Feststellung in Zusammenhang, daß unter den Tieren, deren Namen gleichlautend sind mit den Ausdrücken für Glück, Macht und Reichtum, in China keines so oft abgebildet wird wie gerade die Fledermaus.

$\mathrm{Da}$ nun das in der javanischen Kunst nachweislich seit dem 16. Jahrhundert auftretende Fledermausmotiv zweifellos chinesischen Ursprungs ist, wäre es für die Beziehungsforschung nicht unwichtig, nachzugehen und $\mathrm{zu}$ erfahren, bis in welche Zeit sich die Darstellung der Fledermaus in China selbst zurückverfolgen läßt.

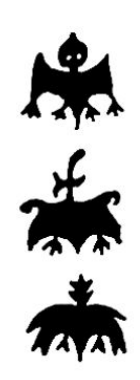

A

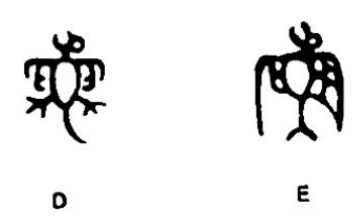

Fig. 3. Bildzeichen für Fledermaus. A, B, C: auf alten Bronzespiegeln, vermutlich aus der Han-Zeit. D. E: auf Kultbronzen vom Typ "Chüeh".

(Nach TAKada Chu-Shu)
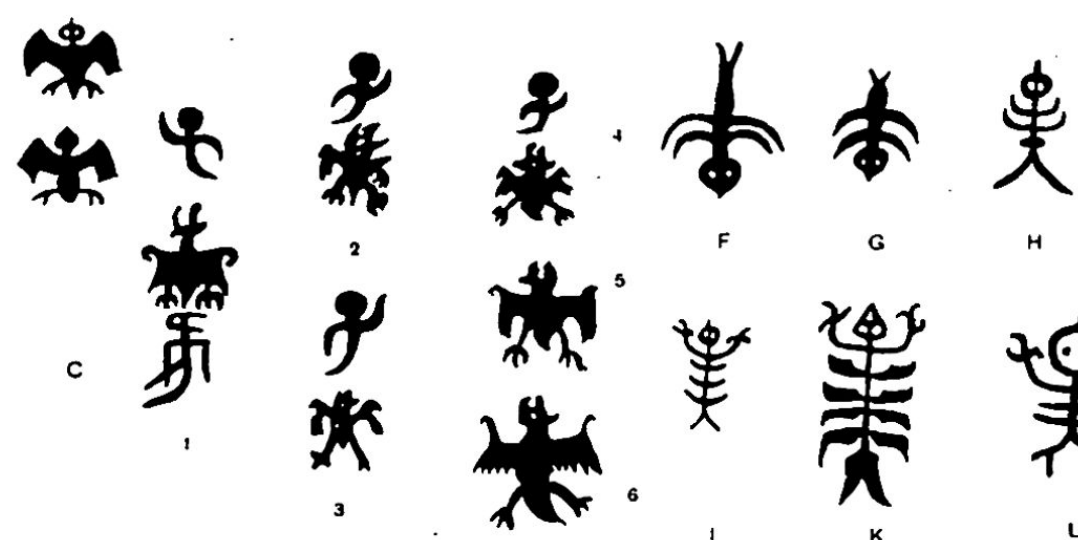

H
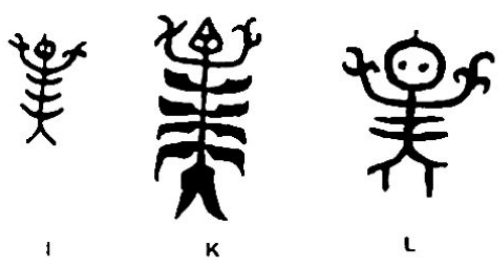

Fig. 4. Bildzeichen für «Fledermaus» auf altchinesischen Sakralbronzen. Nr.1-4 aus der ShangZeit. (Dem Zeichen für «Fledermaus » geht dasjenige für «Sohn» voraus. Nr. 5-6 aus der frühen Chou-Zeit. (Nach Lo CHEN-Yü)
Fig. 5. Altchinesische Schriftzeichen, die irrtümlicherweise als Fledermäuse angeführt worden sind und in Wirklichkeit Zikaden darstellen. (Nach C. HENTZE)

17 Foussagrives: Siling, étude sur les tombeaux de l'ouest de la dynastie des T'sing. Paris 1907.

18 A. SteinmanN: Enkele opmerkingen betreffende de plantornamenten van Mantingan. Djawa, XIV, 1934, S. 89-97.

19 J. Brandes: Driemaandelijksch rapport over April, Mei en Juni 1903. Rapport oudheidk. Commissie in Nederl. Indie, 1903, S. 29-32.

${ }^{20}$ Men schikt de makara's zoo, dat zij tezamen een leeuwenkop geven. Men geeft den leeuwenkop den vorm van een vleermuis en doet dat ook met de het meest op den voorgrond tretende partijen van de makara's." 

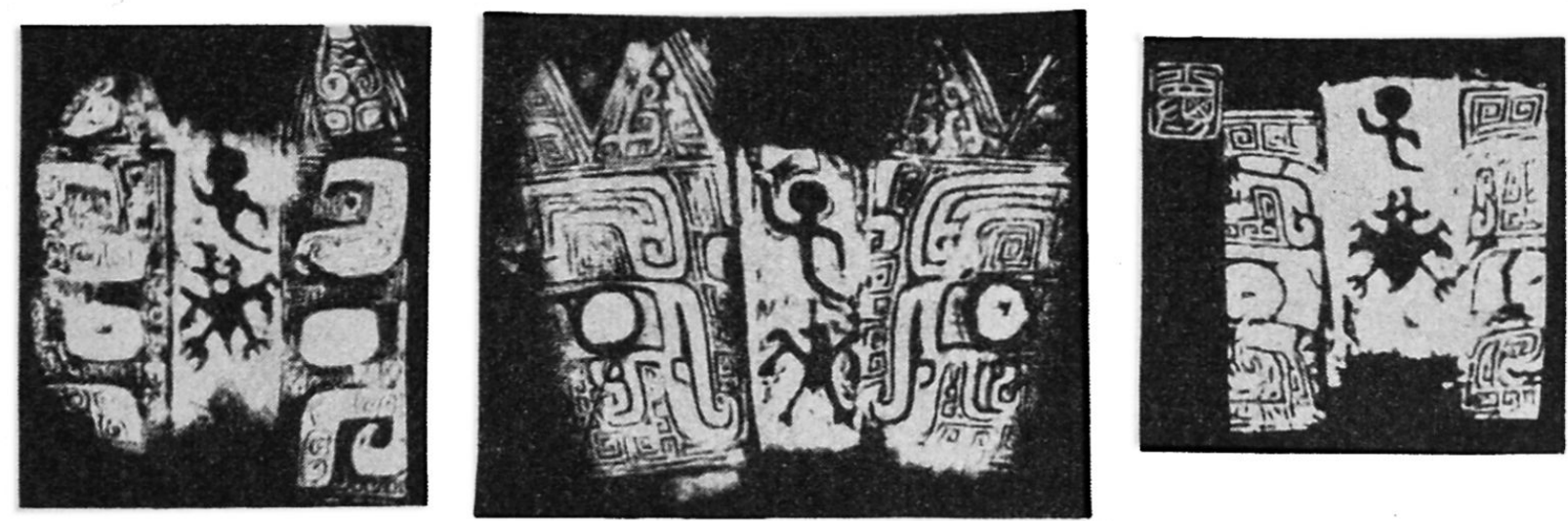

Fig. 6. Abklatsche archaischer Bildzeichen für Fledermaus auf altchinesischen Sakralbronzen (Shangdynastie). - Dem Zeichen für "Fledermaus" geht dasjenige für "Sohn" voraus. (Nach Lo CHEN-Yü)

Bedauerlicherweise ist dieser äußerst interessanten und sich von selbst aufdrängenden Frage durch die hier erwähnten Autoren (Brandes, Lessing, Wirz), die sich mit dem Fledermausproblem beschäftigten, bis jetzt keine Beachtung geschenkt worden. Aus ihren Angaben ließ sich höchstens die Vermutung ableiten, daß die Beliebtheit und allgemeine Verwendung des glückbringenden, auf der Lautähnlichkeit mit "Fu» (= Glück) beruhenden Fledermausmotivs in China relativ jungen Datums sein dürfte. Noch LAUfer ${ }^{21}$ war der Ansicht, daß sich die Fledermaus als Glückssymbol auf Jadegegenständen nicht früher als bis zur T'ang-Periode (618-906) in China mit Sicherheit nachweisen lasse. Später konnte jedoch TAKADA $\mathrm{CHUSHU}^{22}$ an Hand seiner Untersuchungen alter Schriftzeichen auf Kultgefäßen und Bronzespiegeln feststellen, daß das Zeichen für Fledermaus bereits zur Zeit der Han-Dynastie (206 v. Chr. bis $220 \mathrm{n}$. Chr.) bekannt war und damals das Wortspiel Fu (Fledermaus) = Fu (Glück) bereits eine Rolle spielte (siehe Fig. 3), was auch aus dem Dialektwörterbuch hervorgeht, das EBERHARD dem YANG HsIUNG ${ }^{23}$ zuschreibt. Während nun das auf die Fledermaus bezügliche Lautrebus-Wortspiel in China bis zum ersten vorchristlichen Jahrhundert mit Bestimmtheit nachweisbar ist, geht das Vorkommen des chinesischen Bildzeichens für die Fledermaus viel weiter zurück! Prof. Dr. CARL Hentze machte mich freundlicherweise darauf aufmerksam, daß im großen, mir leider nicht zugänglichen Werk von Lo CHEN-Y نّ̈ Inschriften von Bronzegefäßen aus der frühen Chou- und selbst aus der Shang-Zeit abgebildet sind, die das Bildzeichen für Fledermaus in Verbindung mit dem daran vorausgehenden Zeichen für "Sohn» zeigen (Fig. 4 und 6), von denen TAKADA annimmt, daß sie ebenfalls zum obengenannten Wortspiel oder Lautrebus in Beziehung stehen könnten. Da indessen auf diesen Inschriften das Zeichen «Sohn» mit nachfolgendem Tierbild, das neben der Fledermaus auch andere Tiere umfassen kann, mehrfach vorkommt, kann es sich nach Ansicht von HENTzE ebensogut um einen Namen handeln ${ }^{25}$. Auf anderen, von Lo CHEN-Y $\ddot{U}$ veröffentlichten Inschriften vcn Bronzegefäßen, die Hentze in die frühe Chou-Zeit versetzt (Fig. 4, Nr. 5-6), kommen Bildzeichen vor, die zweifellos die Fledermaus darstellen. Unter dem Formenschatz der ikonographisch bedeutsamen Tierornamentik, im «Tierstil» frühchinesischer Bronzegefäße der Shang- und Chou-Zeit, kommt die Fledermaus dagegen nicht vor.

21 B. LAUfer: Jade. Field mus. of Natural History, anthrop. series, vol. X, Chicago, 1912, S.168, 287-289.

22 Takada Chushu: Ku chou p'ien (jap. Ko-chu-hen), Tokyo, 97, 1925, S. 28 verso.

23 Yang Hsiung: Fang Yen (Dialektwörterbuch), 53 v. Chr. bis $18 \mathrm{n}$. Chr. Die Autorschaft von Yang Hsiung wird allerdings von Giles (Biographical Dictionary) angezweifelt.

24 Lo Chen-Yü: San tai chi chin wen ts'un. Mukden, gedruckt 1937. Vol. XIV und XV, S. 28 und 29.

25 Herrn Prof. Dr. C. Hentze möchte ich hier für seine wertvolle Mitarbeit und die freundliche Übermittlung der Abklatsche herzlich danken. 
Nach einer brieflichen Mitteilung von Prof. Hentze spielt die Fledermaus in der kultischen Ikonographie der altchinesischen Bronzen, wie eine Durchsicht von Tausenden von Gegenständen ergab, überhaupt keine Rolle. Einige der von LEROI-GourhaN ${ }^{26}$ als Fledermausdarstellungen angesehenen Tierformen haben sich als Zikaden erwiesen (siehe R. Grousset: L'évolution des bronzes archaïques Paris, 1937, Fig. S. 13), und dasselbe gilt für die auf Fig. 5 abgebildeten altertümlichen Schriftzeichen, die TAKADA irrtümlicherweise fürFledermausdarstellungen hielt; nachdem NAKASHIMA ${ }^{27}$ bereits Zweifel an dieser Identifizierung geäußert hatte, konnte HENTZE ${ }^{28}$ nachweisen, daß es sich um Zi-
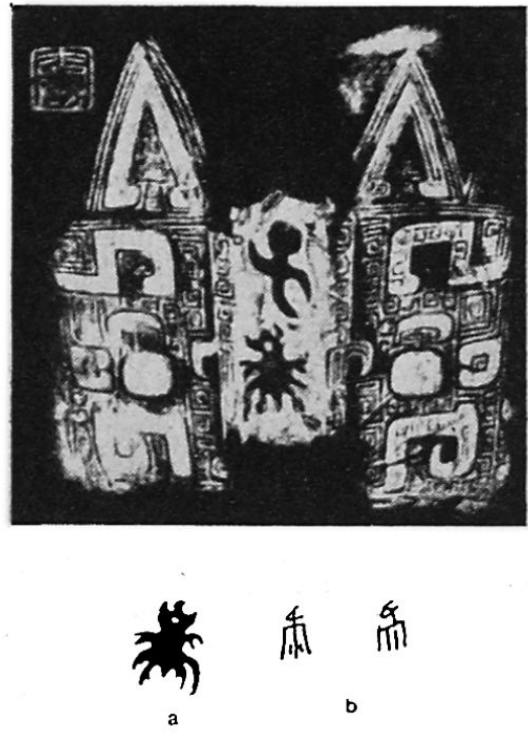

Fig. 7. Bildzeichen für "Fledermaus" aus der Zeit der Shang-Dynastie. Oben: Abklatsch einer Inschrift auf einem Bronzegefäß. (Nach Lo CHEN-Yü). Unten: Bildzeichen der Fledermaus als Ortsbezeichnung (Knochenschrift). kaden handelt. Die Zeichen für Fledermaus, die man auf Schildkrötenschalen-Inschriften und auf Knochenschriften antrifft, sind Ortsbezeichnungen, die allerdings heute nicht mehr vorkommen (Fig. 7), während es sich bei anderen Fledermauszeichen, schon in früher Zeit bis zur Han-Dynastie, auch um Personennamen handeln kann.

Dem Fang-Yen (Dialektwörterbuch) des Yang Hsiung (53 v. Chr. bis $18 \mathrm{n}$. Chr.) lassen sich, wie mir Prof. Hentze mitteilt, folgende Dialektnamen für die Fledermaus entnehmen: Westlich des Grenzgebietes Fu-i, Fei-shu (geflügelte Ratte), Lao-shu (alte oder kluge Ratte) und Hsien-shu (Ratte der Genien oder Unsterblichen). Innerhalb des Grenzgebietes und im Westen: Pien-fu als der gebräuchlichste Name (wobei Pien in keiner einzigen sehr alten Form vorkommt, sondern nur das Zeichen Fu); in der nördlichen Landschaft Yen heißt die Fledermaus Shih-me.

Es ergibt sich daraus die Feststellung, daß das Bildzeichen für Fledermaus als Ortsbezeichnung und Personennamen in China wohl sehr alt ist, daß dagegen die auf ihrem Gleichklang mit dem chinesischen Wort für Glück beruhende Darstellung der Fledermaus als Wortspiel höchstens bis zum ersten vorchristlichen Jahrhundert nachgewiesen werden kann. In der indonesischen Kunst dagegen tritt das von China aus importierte Fledermausmotiv, soweit sich bisher feststellen ließ, erst viel später, nämlich im 16. Jahrhundert, auf Java in Erscheinung.

\section{LA CHAUVE-SOURIS DANS LA RELIGION, LE FOLKLORE ET L'ART DE L'INDONÉSIE ET DES PAYS VOISINS}

Parmi les conceptions magico-religieuses des peuples de l'Indonésie, la chauve-souris joue un rôle spécial. Tantôt elle est redoutée comme un animal de mauvaise augure, tantòt elle est associée aux mythes d'origine ou bien elle apparaît comme animal totémique.

Par contre, son apparition dans l'art indonésien, ou elle ne joue d'ailleurs qu'un rôle tout à fait secondaire, n'a aucun rapport avec les croyances auxquelles elle est associée. En Indonésie, la plupart des figurations de chauve-souris sont d'origine chinoise.

Quoique le pictogramme de la chauve-souris apparaisse déjà sous la dynastie des Shang, ce n'est que vers le premier siècle avant J.-Chr., sous la dynastie des Han, que les plus anciennes représentations de la chauve-souris basées sur l'homophonie avec le mot $\mathrm{Fu}$ (= bonheur) font leurs apparition.

\section{IL PIPISTRELLO NELLA RELIGIONE, NEI COSTUMI E NELL'ARTE DELL'INDONESIA E DEI PAESI LIMITROFI}

Il pipistrello assume un posto particolare nelle concezioni magico-religiose dei popoli indonesiani: talora è apportatore di disgrazia, tal'altra è considerato totem. Nell'arte rimane invece in un piano di second'ordine: la maggior parte delle rappresentazioni, = di cui le più antiche risalgono al XVIo secolo, proviene dalla Cina, paese nel quale l'imagine del pipistrello appare già sotto la dinastia di Shang, e connesso alla parola "felicità » solo verso il primo secolo a. C., sotto la dinastia di Han.

${ }^{26}$ A. Leroi-Gourhan: Bestiaire du bronze chinois de style Tscheou. Paris, 1936, S. 20-22, "La chauve-souris». ${ }^{27}$ Nakashima: Shu-ch'ieh yüan-yüan, Tokyo 1934, II, S. 202/203.

${ }^{28}$ C. Hentze: Frühchinesische Bronzen und Kultdarstellungen, Antwerpen 1937, Textband, S. 19. 